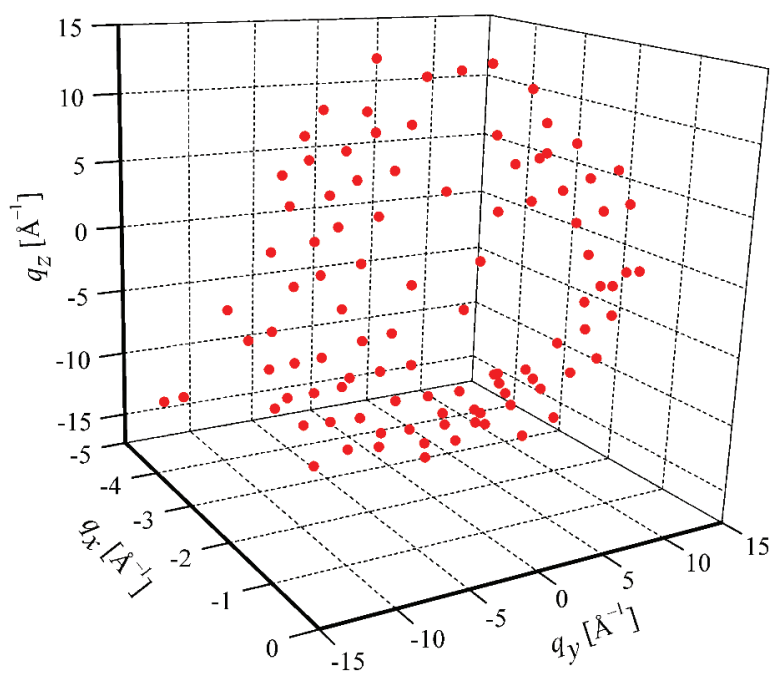

Figure 1. The recorded Laue pattern of GaAs after resolving the Laue spots' energies and converting the three-dimensional information into reciprocal-space coordinates. 101 Bragg peaks were collected in a single X-ray shot occupying reciprocal lattice points of GaAs in the defined coordinate system.

Keywords: energy-dispersive Laue diffraction, X-ray structure analysis, $\mathrm{X}$-ray spectroscopy, white synchrotron radiation, $\mathrm{pnCCD}$

\section{MS39-P13 The DIALS framework}

James M. Parkhurst ${ }^{1,2}$, Graeme Winter ${ }^{1}$, David Waterman ${ }^{3}$, Richard Gildea ${ }^{1}$, Luis Fuentes-Montero ${ }^{1}$, Gwyndaf Evans ${ }^{1}$

1. Diamond Light Source

2. MRC LMB

3. $\mathrm{CCP} 4$

email: james.parkhurst@diamond.ac.uk

Recent technological advances and changes in methodology in X-ray crystallography, such as high frame-rate pixel array detectors and serial femtosecond crytallography have created a need for the developement of new algorithms; however, current integration programs for X-ray diffraction data are limited by their lack of openness or extensibility, resulting in a barrier between the developement of new integration algorithms and their deployment on the beamline.

Here we present the DIALS (diffraction integration for advanced light sources) framework and integration package which is both a standalone application for the integration of X-ray diffraction data and a modular framework for developing new integration programs. The aim of the DIALS framework is to provide an extensible architecture within which new integration algorithms may be developed and quickly deployed without developers being required to write and maintain large amounts of high-level application source code. Rather, it is the aim of DIALS to liberate developers to allow them to concentrate soley on the scientific content of the algorithms themselves.

The DIALS framework implements a simple plugin system that allows algorithms to be loaded at runtime to perform certain tasks such as spot finding, indexing, refinement, background subtraction and integration. At the cost of writing a small amount of Python code, the developer can add an algorithm that can be loaded and selected at runtime without any modification of the DIALS framework itself. This design allows DIALS to be flexible with respect to the experiment being performed. For example, different algorithms can be used for data obtained using the rotation method or serial femtosecond crystallography.

DIALS provides a comprehensive suite of command line applications for the analysis of X-ray diffraction data as well as providing tools for visual inspection of the raw data and processing results. DIALS is written in a mixure of Python and $\mathrm{C}++$ and is available for download from http://sourceforge.net/projects/dials; the source code is available under an open-source BSD license.

Keywords: Integration, data processing, X-ray crystallography, software 